\section{Kompass \\ Ophthalmologie}

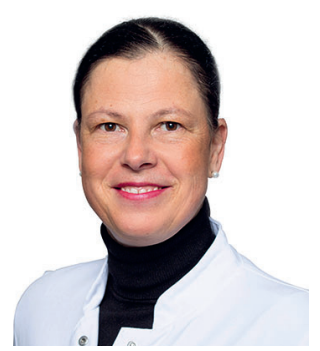

Nicole Stübiger

Klinik- und Poliklinik für Augenheilkunde, Universitätsklinikum Hamburg-Eppendorf, Hamburg, Deutschland

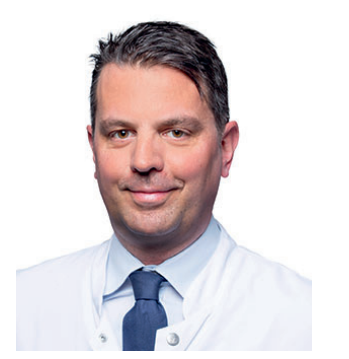

\section{Stephan Johannes Linke}

zentrumsehstärke, Augenarztpraxis am UKE, Klinik- und Poliklinik für Augenheilkunde, Universitätsklinikum Hamburg-Eppendorf, Hamburg, Deutschland

\title{
Das Trockene Auge: komplexe Herausforderung für den Augenarzt
}

Die TFOS - Tear Film \& Ocular Surface Society - definierte 2017 in ihrem zweiten Dry Eye Workshop (DEWS II) das Trockene Auge als eine multifaktorielle Störung der Tränen und der Augenoberfläche, die durch den Verlust der Homöostase des Tränenfilms charakterisiert ist und mit okulären Beschwerden einhergeht, bei denen Tränenfilminstabilität, Hyperosmolarität, Entzündung sowie Beschädigung der Augenoberfläche und auch neurosensorische Abnormalitäten eine wichtige ätiologische Rolle spielen [1]. Vier pathogenetische Schlüsselmechanismen spielen also bei der Erkrankung Trockenes Auge eine zentrale Rolle: Tränenfilminstabilität, Tränen-Hyperosmolarität, Apoptose und Entzündung. Ohne Intervention verstärken sich diese stetig im Sinne eines «Circulus vitiosus», mit der Folge einer Zunahme der klinischen Zeichen und meist auch der subjektiven Symptomatik [2].

Die dokumentierte Prävalenz dieser häufigen Augenerkrankung liegt weltweit zwischen 5,5 und 57,1\% [3-6]. In Deutschland leiden etwa 15-17\% der Bevölkerung an einem Trockenen Auge [5, 7]. Die Erkrankungshäufigkeit nimmt mit steigendem Alter zu, wobei Frauen häufiger als Männer betroffen sind [3, 7]. Etwa jeder 3. Patient in einer Augenarztpraxis leidet an einem Trockenen Auge.
Beim Trockenen Auge, welches synonym auch als Dry Eye bzw. als Sicca-Syndrom bezeichnet wird, lassen sich im Wesentlichen zwei Formen unterscheiden: das hypovolämische Trockene Auge, das aufgrund einer Mindersekretion der wässrigen Phase, also durch ein zu geringes Tränenvolumen, entsteht, und das hyperevaporative Trockene Auge, welches auf eine Störung der Lipidphase und die dadurch verursachte verstärkte Verdunstung zurückzuführen ist. Das Trockene Auge aufgrund einer Lipid-Störung tritt häufiger auf als das Trockene Auge aufgrund eines wässrigen Defizites. Meist liegt jedoch eine Mischform vor [8-10].

Das Trockene Auge stellt den Ophthalmologen aufgrund seiner Komplexität nicht selten vor große Herausforderungen: Die Symptomatik und das klinische Bild sind häufig uneinheitlich, für Diagnostik und Therapie stehen unzählige verschiedene Ansätze zur Verfügung [10], aus denen die für den individuellen Patienten geeignetsten Lösungen ausgewählt werden müssen und - last but not least - gelten Patienten mit Trockenem Auge vielfach als schwierige Patienten, denen der Augenarzt trotz limitierter Ressourcen und fehlender Kostenübernahme durch die Krankenkassen gerecht werden muss.

Im Rahmen dieser Ausgabe von Kompass Ophthalmologie mit dem Schwerpunkt information@karger.com www.karger.com/kop

(c) 2020 S.Karger GmbH, Freiburg

Karger ${ }^{\prime \prime}=$
Prof. Dr. Nicole Stübiger \& Prof. Dr. Stephan Johannes Linke

Klinik- und Poliklinik für Augenheilkunde

Universitätsklinikum Hamburg-Eppendorf

Martinistraße 52, Hamburg, Deutschland

n.stuebiger@uke.de; slinke@uke.de 
Trockenes Auge sollen 2 Publikationen vorgestellt werden, die zahlreiche aktuelle Aspekte zu Ätiologie, Diagnostik, Therapie und Patientenführung in prägnanter Form näher beleuchten. Wir hoffen, mit der Aus-

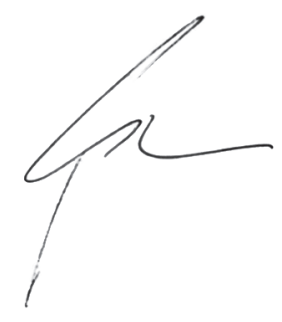

Prof. Dr. Nicole Stübiger wahl dieser Beiträge den Lesern einen aktuellen Überblick sowie einen anschaulichen Einblick in ein spannendes Feld der Augenheilkunde bieten zu können.
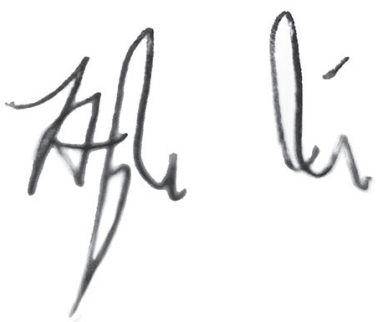

Prof. Dr. Stephan Johannes Linke

\section{Literatur}

$\checkmark 1$ Craig JP, Nichols KK, Akpek EK, et al.: TFOS DEWS II Definition and Classification Report. Ocul Surf. 2017;15:276-283.

$\checkmark 2$ Baudouin C, Aragona P, Messmer EM, et al.: Role of hyperosmolarity in the pathogenesis and management of dry eye disease: proceedings of the OCEAN group meeting. Ocul Surf. 2013;11:246--258.

3 Stapleton F, Alves M, Bunya VY, et al.: TFOS DEWS II Epidemiology Report. Ocul Surf. 2017;15: 334-365.

$\checkmark 4$ Viso E, Rodriguez-Ares MT, Gude F: Prevalence of and associated factors for dry eye in a Spanish adult population (the Salnes Eye Study). Ophthalmic Epidemiol. 2009;16: 15-21.

5 Reitmeir P, Linkohr B, Heier M, et al.: Common eye diseases in older adults of southern Germany: results from the KORA-Age study. Age Ageing. 2017;46:481-486. 